\title{
Reliability Assessment of Pressurized Pipes with Inclined Defects
}

\author{
Yanlin Wang ${ }^{1, \mathrm{a}}$, Weigang Wang ${ }^{1, \mathrm{~b}}$, Wei Yang ${ }^{2}$ and Chun-Qing $\mathrm{Li}^{1, \mathrm{c}}$ \\ ${ }^{1}$ School of Engineering, RMIT University, Melbourne, 3001, Australia, as3733192@student.rmit.edu.au, \\ bweigang.wang@rmit.edu.au, ${ }^{\circ}$ chunqing.li@rmit.edu.au \\ ${ }^{2}$ Faculty of Architecture, Design and Planning, The University of Melbourne, Melbourne, 3010, \\ Australia,wei.yang@unimelb.edu.au
}

\begin{abstract}
Inclined defects in pipelines can lead to failures in the form of mixed mode fracture. A review of the literature suggests that few studies have been carried out on reliability assessment of pipelines considering mixed mode fracture. This paper intends to present a reliability-based method for assessing fracture failures of pressurized pipes with inclined defects. Richard's criterion is employed in the development of the limit state function for reliability assessment. A Stochastic model of load effects is developed, and first passage method with a developed analytical solution is used to predict the pipe failures. A worked example is provided to illustrate the application of the proposed method. The method presented in this paper can help pipe engineers and asset managers to develop a reliability-based maintenance strategy for better management of pipelines.
\end{abstract}

Keywords: Pressurized Pipes, Inclined Crack, Mixed Mode Fracture, Time-Dependent Reliability, First Passage Probability.

\section{Introduction}

Pipelines are widely used as one of the safest and most economical structures to transport gas or liquid (e.g., water; natural gas) in industries (Keshtegar and Miri, 2014). However, most of them have been in service for a long time, and the exposure to the corrosive environment increases the likelihood of failures before the end of the intended design life (Carbal and Kimber, 1997). The consequences of pipeline failures can be economically, socially, and environmentally devastating (Mahmoodian and Li, 2017). Therefore, it is essential to fully understand the failure mechanisms of pipes and accurately predict its service life.

A literature review suggests that most of the previous researches on the pipe failure assessment mainly considered the loss of strength due to the reduction of wall thicknesses of pipes (e.g., Ahammed and Melchers, 1997; Mahmoodian and Li, 2017; Liu et al., 2019). Nevertheless, it has been found that pipe failures are more likely to be fracture type due to the stress concentration near the tips of crack-like defects (Li and Mahmoodian, 2013; Fu et al., 2019). As the cracked structures often fail unexpectedly at the applied stress much below the material strength (Anderson, 2005), it is necessary to assess the integrity of pipeline based on fracture mechanics. Also, the defects on the pipe walls are often caused by corrosion, which can grow in any directions depending on the heterogeneity of surrounding soil properties (Wang et al., 2018; Wang et al., 2019). Moreover, the crack-like defects in pipes most likely grow in an inclined manner because of the complex stress state (Li et al., 2016). As a result, in these pipes (e.g., Figure 1), the failures can be induced by a mixed mode loading interaction. Some previous researches (e.g., by Erdogan and Shih, 1968; Chang et al., 2006) indicated that the single mode fracture failure criterion is seldom appropriate for assessing the integrity of structural components with inclined cracks. Thus, a method based on mixed mode fracture criteria is more appropriate to assess the fracture failures of pipes. To predict the pipe failures, time-dependent reliability methods have been successfully used since the parameters associated with pipe 
failures are highly uncertain and changing with time ( $\mathrm{Fu}$ et al., 2019). Although several researchers, e.g., Li and Mahmoodian (2013); Fu et al., (2019); Wang et al., (2019), have assessed the probability of pipe failures using time-dependent reliability methods for different defect types (e.g., sharp corrosion pit; elliptical corrosion pit), these works only focuses on Mode I (opening mode) fracture failure. The reliability assessment of pipelines with inclined defects considering mixed mode fracture, in comparison, is highly limited.

This paper presents a time-dependent reliability method for pressurized pipe based on first passage probability theory. The cast iron pipe with an external inclined crack-like defect is considered in this paper. A stochastic model of the stress intensity factor is developed by incorporating Richard's criterion into the limit state function. A case study is presented to illustrate the proposed method. The proposed method can be applied to the risk-based maintenance strategy for pipelines.
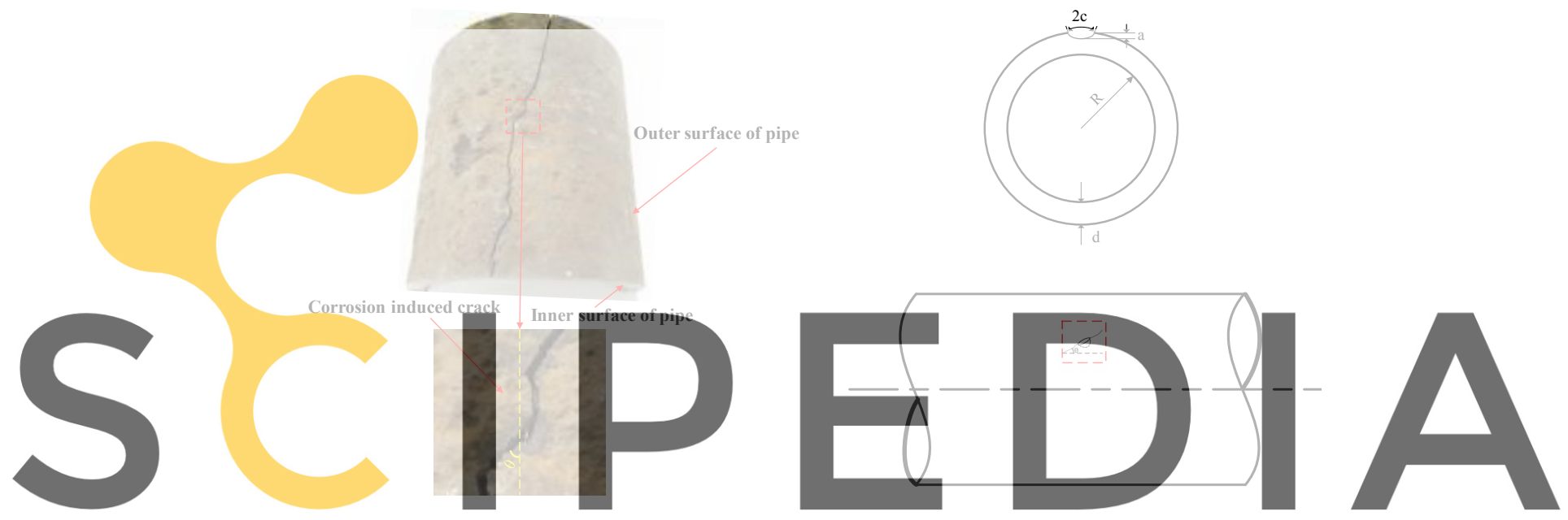

Register for free at https//Figure 1. A pressurized pipe with inclined external surface defects.jithout the watermark

\section{Problem Formulation}

To determine the probability of failure of a pipe, a failure criterion must be established. In the structural reliability theory, the criterion can be expressed in the form of a limit state function as follows (Melchers, 1999):

$$
G(L, R, t)=L(t)-R(t)
$$

where $L(t)$ is the load effect on the structures at time $t ; R(t)$ is the acceptable limit (resistance) at time $t$. As both load effect $(L(t))$ and acceptable limit $(R(t))$ are time-dependent, all or some of the basic variables should be modelled as stochastic processes. For reliability problems involving the stochastic processes, the structural failure event is that the stochastic processes of the load action process $L(t)$ initially pass upwards an acceptable limit (threshold) $R(t)$ during the service life. With the assumption of Poisson processes, the probability of failure can be determined as follows (Melchers, 1999):

$$
P_{f}(t)=1-\left[1-P_{f}(0)\right] e^{-\int_{0}^{t} v d t}
$$

where $P$ indicates the probability of an event; $P_{f}(0)$ is the probability of failure at time $t=0$ and $v$ is the mean rate for the load action process $L(t)$ to cross upwards the threshold $R(t)$. The up-crossing rate $v$ can be determined by the Rice formula (Rice, 1944) 


$$
v=v_{R}^{+}=\int_{\dot{R}}^{\infty}(\dot{R}-\dot{L}) f_{L \dot{L}}(L, \dot{R}) d \dot{L}
$$

where $v_{R}^{+}$is the up-crossing rate of the load action process $L(t)$ relative to the threshold $R(t)$; $\dot{R}$ is the slope of $R$ with respect to time; $\dot{L}$ is the time derivative process of $L ; f_{L \dot{L}}$ is the joint probability density function for $L$ and $\dot{L}$. Li and Melchers (1993) derived an analytical solution to Equation (3) when $L(t)$ is a Gaussian process and the threshold $\mathrm{R}$ is deterministic. It is expressed as follows:

$$
v_{R}^{+}=\frac{\sigma_{\dot{L} \mid L}}{\sigma_{L}} \phi\left(\frac{R-\mu_{L}}{\sigma_{L}}\right)\left\{\phi\left(\frac{\dot{R}-\mu_{\dot{L} \mid L}}{\sigma_{\dot{L} \mid L}}\right)-\frac{\dot{R}-\mu_{\dot{L} \mid L}}{\sigma_{\dot{L} \mid L}} \Phi\left(-\frac{\dot{R}-\mu_{\dot{L} \mid L}}{\sigma_{\dot{L} \mid L}}\right)\right\}
$$

where $\mu$ and $\sigma$ are the mean and standard deviation of random variables represented by subscripts $L$ and $\dot{L} ;$ ' $\mid$ ' denotes the condition; $\phi$ and $\Phi$ indicate the standard normal density and distribution functions. For a given Gaussian stochastic process with mean function $\mu_{L}(t)$ and auto-covariance function $C_{L L}\left(t_{i}, t_{j}\right)$, all terms in Equation (4) can be determined as follows:

$$
\begin{gathered}
\mu_{\dot{L} \mid L}=E[\dot{L} \mid L=R]=\mu_{\dot{L}}+\rho_{L} \frac{\sigma_{\dot{L}}}{\sigma_{L}}\left(R-\mu_{L}\right) \\
\sigma_{\dot{L} \mid L}=\left[\sigma_{L}^{2}\left(1-\rho_{L}^{2}\right)\right]^{1 / 2}
\end{gathered}
$$
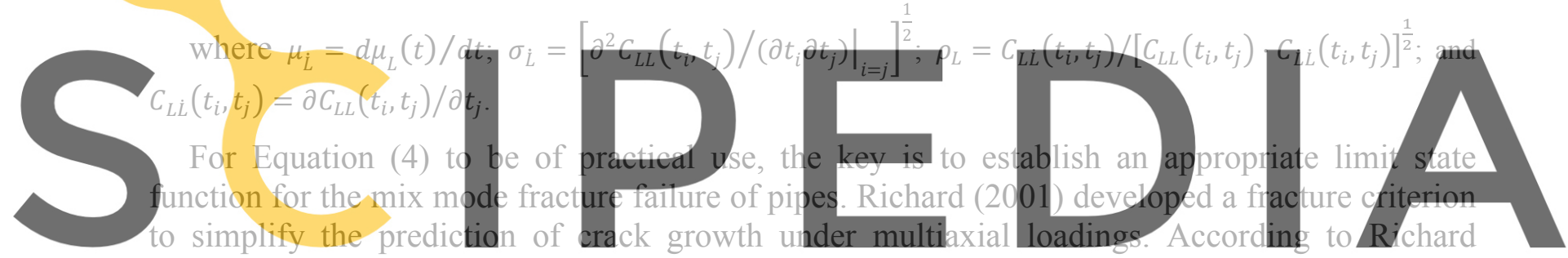

(2001), unstable crack growth in brittle materials would occur if the local loading condition

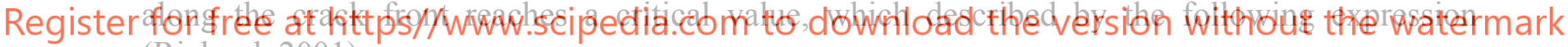
(Richard, 2001):

$$
K_{v}=\frac{K_{I}}{2}+\frac{1}{2} \sqrt{K_{I}^{2}+4\left(\alpha_{1} K_{I I}\right)^{2}+4\left(\alpha_{2} K_{I I}\right)^{2}} \geq K_{I C}
$$

where $K_{v}$ is an equivalent stress intensity factor; $K_{I}, K_{I I}$, and $K_{I I I}$ are the Mode I (opening mode), Mode II (in-plane shear mode), and Mode III (out-of-plane shear mode) stress intensity factors, respectively; $K_{I C}$ is the Mode I fracture toughness which quantifies the material resistance to crack extension; $\alpha_{1}=K_{I c} / K_{I I c}$ and $\alpha_{2}=K_{I c} / K_{I I I c}$ with $K_{I I c}$ as the fracture toughness for pure Mode II and $K_{I I I C}$ as the fracture toughness for pure Mode III. With $\alpha_{1}=$ 1.155 and $\alpha_{2}=1.0$, Equation (6) was found having an excellent agreement with other criteria (e.g., developed by Schöllmann et al., 2002) and the experimental data in brittle materials (Richard et al., 2014). Based on Richard's criterion, Equation (1) can be expressed as:

$$
G(t)=\frac{K(t)_{I}}{2}+\frac{1}{2} \sqrt{K(t)_{I}^{2}+5.3361 K(t)_{I I}^{2}+4 K_{I I I}^{2}}-K(t)_{I C}
$$

The determination of stress intensity factors is presented in the next section.

\section{Model of Load Effects}

The stress intensity factor $(K)$ represents the magnitude of the stress fields surrounding the tip of crack or crack-like defect. Its magnitude depends on the far-field stress level $\sigma$, the size of crack $a$, and the geometries of the bodies. For a pipe with an external inclined defect as shown in 
Figure 1, the stress intensity factor $K$ can be generalised as follows (Li et al., 2016):

$$
\boldsymbol{K}=\frac{p R}{d} \sqrt{\pi \frac{a}{Q}} \boldsymbol{F}\left(\frac{a}{d}, \frac{a}{c}, \frac{d}{R}, \xi, \theta\right)
$$

where $\boldsymbol{K}=\left\{K_{I}, K_{I I}, K_{I I I}\right\}^{T} ; p$ is the internal pressure; $p R / d$ is the average hoop stress in pipes; $\xi$ is used to define the position of an arbitrary point along the semi-elliptical crack; $\theta, c, d$, and $R$ are defined in Figure 1; $Q$ is the shape function which can be expressed as follows (Shiratori and Miyoshi, 1986):

$$
Q=1+1.464\left(\frac{a}{c}\right)^{1.65} ; \frac{a}{c} \leq 1
$$

In Equation (8), $\boldsymbol{F}\left(\frac{a}{d}, \frac{a}{c}, \frac{d}{R}, \xi, \theta\right)=\left\{F_{I}, F_{I I}, F_{I I I}\right\}^{T}$, where $F_{I}, F_{I I}$, and $F_{I I I}$ are the influence coefficient functions for Mode I, II, and III, respectively. Li et al., (2016) have developed the influence coefficients of stress intensity factors for inclined cracks in pipes under internal pressure using three-dimensional finite element analysis. The approximations of the influence coefficients can be expressed as follows:

$$
\begin{gathered}
F_{I}=M_{I} N_{I} \sin ^{2}\left(h_{I}^{11}+h_{I}^{12} \theta\right) \\
F_{I I}=M_{I I}\left[h^{6}(\xi)+h^{7}(\xi)^{3}\right] \sin \left(h_{I I}^{8}+h_{I I}^{9} \theta\right)
\end{gathered}
$$

where $k$ and $n$ are empinical coefficients to be determined from field data.

Apart from the growth of corrosion pit, the operating internal pressure and pipe wall thickness also change with time. Therefore, it is justifiable to model the load effect $L(t)$ as a stochastic process. The randomness of the load effect can be taken into account by introducing a random variable, $\xi_{L}$. This variable is defined in such a way that its mean is unity, i.e., $E\left(\xi_{L}\right)=1$, and its coefficient of variation, $\lambda_{L}$, is a constant (Mahmoodian and Li, 2017). The load effect can be expressed as follows:

$$
L(t)=L_{c}(t) \cdot \xi_{L}
$$

where $L_{c}(t)$ is treated as a pure time function determined by the load effect function. The mean and auto-covariance functions of $L(t)$ can be expressed as follows ( $\mathrm{Li}$ and Melchers, 2005):

$$
\begin{gathered}
\mu_{L}(t)=E[L(t)]=L_{c}(t) \cdot E\left[\xi_{L}\right]=L_{c}(t) \\
C_{L L}\left(t_{i}, t_{j}\right)=\lambda_{L}^{2} \rho_{L} L_{c}\left(t_{i}\right) L_{c}\left(t_{j}\right)
\end{gathered}
$$

where $\rho_{L}$ is the auto-correlation coefficient for $L(t)$ between two points in time $t_{i}$ and $t_{j}$. 
Table 1. Values of $h$ in Equation (10) with $d / R=0.1$ and $a / c=0.4$ (Li et al., 2016).

\begin{tabular}{cccc}
\hline Constant & $\mathbf{i}=\mathbf{I}$ & $\mathbf{i}=\mathbf{I I}$ & $\mathbf{i}=\mathbf{I I I}$ \\
\hline $\mathrm{h}_{\mathrm{i}}^{1}$ & 0.957 & -0.427 & 0.742 \\
$\mathrm{~h}_{\mathrm{i}}^{2}$ & 0.504 & -0.123 & 0.076 \\
$\mathrm{~h}_{\mathrm{i}}^{3}$ & 0.256 & 0.114 & -0.070 \\
$\mathrm{~h}_{\mathrm{i}}^{4}$ & 0.155 & -0.019 & 0.073 \\
$\mathrm{~h}_{\mathrm{i}}^{5}$ & 0.088 & -0.346 & 0.309 \\
$\mathrm{~h}_{\mathrm{i}}^{6}$ & 0.983 & 0.454 & 0.618 \\
$\mathrm{~h}_{\mathrm{i}}^{7}$ & -0.059 & 0.186 & -0.101 \\
$\mathrm{~h}_{\mathrm{i}}^{8}$ & -0.044 & 3.142 & -0.604 \\
$\mathrm{~h}_{\mathrm{i}}^{9}$ & 0.023 & -2.001 & 1.279 \\
$\mathrm{~h}_{\mathrm{i}}^{10}$ & -0.166 & & -1.016 \\
$\mathrm{~h}_{\mathrm{i}}^{11}$ & 1.589 & & 1.997 \\
$\mathrm{~h}_{\mathrm{i}}^{12}$ & 0.994 & & \\
\hline
\end{tabular}

\section{Worked Example}

The proposed methodology is applied to a case study in which a cast iron pipe with inclined corrosion pits is considered. To calculate the stress intensity factors, the influence coefficient functions for Mode I, II, and III are determined by means of the constants in Table 1 and the assumption of aspect ratio $a / c=0.4$. A cording

Mean

C.O.V

Reference

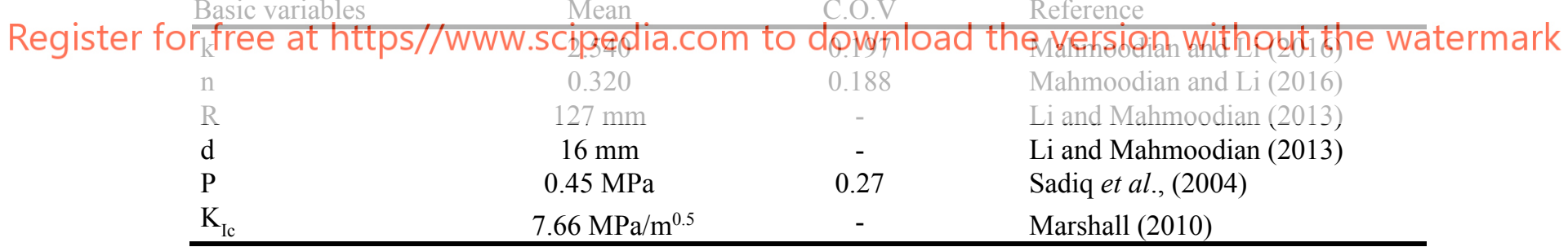

The load effect is calculated by Equation (8), (9) and (10). The mean of function $\mu_{L}$ and standard deviation $\sigma_{L}$ can be calculated as a function of time using Monte Carlo simulation together with the proposed model of pit depth. The up-crossing rate $v_{R}^{+}$can be obtained from Equation (5) for a given auto-correlation coefficient, and the probability of failure can be calculated by Equation (2). 


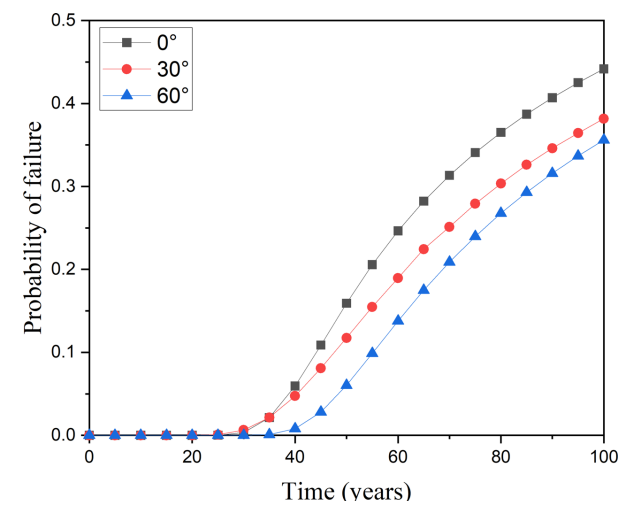

(a)

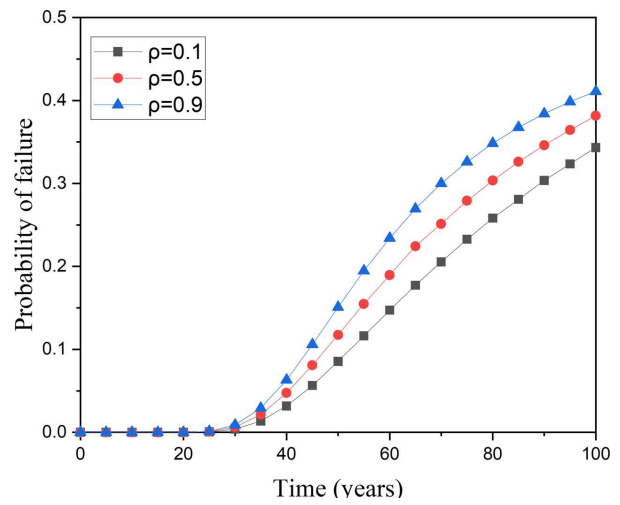

(b)

Figure 2. Probability of pipe failure: (a) for different inclination angles with $\rho \rho=0.5$; (b) for different autocorrelation coefficients with $\theta \theta=30^{\circ}$.

The calculation results of the probability of failure with different inclination angles and autocorrelation coefficients are shown in Figure 2. The probability of pipe failure increases with the exposure time due to the growth of pit depth. Also, it can be seen that the probability of failure decreases with the increase of the inclination angles since the equivalent stress intensity factor $K K$

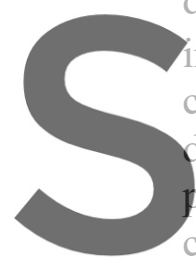
coefficients affect the lepends on many factors. pit depth, are interrelate correlated in the time
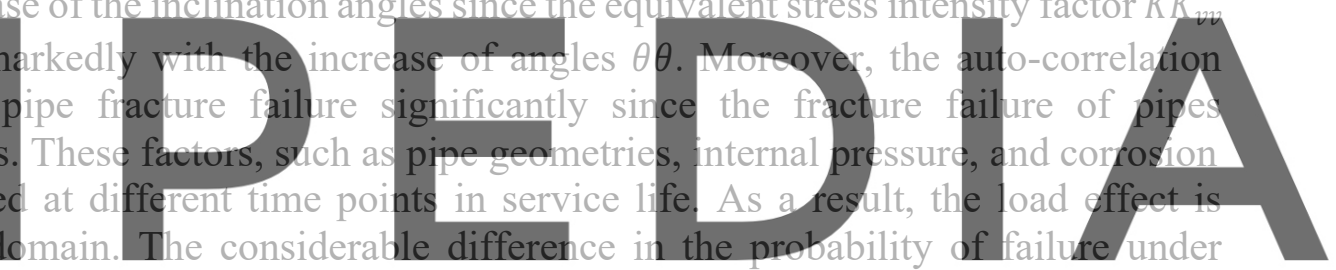

different $\rho \rho$ justifies the necessity of using a time-dependent reliability method based on the

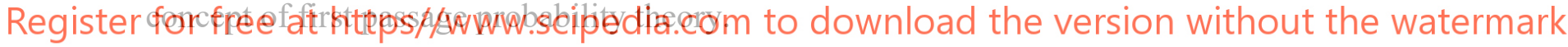

\section{Conclusion}

In this study, the time-dependent failure probability of pipes with inclined crack-like defects was calculated, in which a mixed mode fracture criterion was used to establish the limit state function. First passage probability theory was employed to predict the pipe failures. A case study was provided to illustrate the proposed method. From the results of the case study, it has been found that the stress intensity factors increase rapidly with the increase of corrosion pits depth, and the probability of pipe failure increases for longer service time. Moreover, the probability of failure is highly sensitive to both the auto-correlation coefficients and inclination angles. The smaller the inclination angle is, the higher the probability of failure. As such, for engineering assessment of pipes with inclined defects, more attention should be paid to the defects with smaller inclination angles. The proposed method can be applied to the development of rehabilitation strategies for existing pipe networks. 


\section{Acknowledgements}

Financial support from the Australian Research Council under DP140101547, LP150100413 and DP170102211, and National Natural Science Foundation of China with Grant No. 51820105014 is gratefully acknowledged.

\section{ORCID}

Yanlin Wang: https://orcid.org/0000-0002-4589-9975

Dr. Weigang Wang: https://orcid.org/0000-0002-5803-3572

Dr. Wei Yang: https://orcid.org/0000-0002-3673-6488

\section{Reference}

Ahammed, M. and Melchers, R.E. (1997). Probabilistic analysis of underground pipelines subject to combined stresses and corrosion. Engineering structures, 19(12), 988-994.

Anderson, T.L. (2005). Fracture mechanics: fundamentals and applications. CRC press.

Carbal, M.A. and Kimber, M.J. (1997). Pipeline fracture experiences in Australia and North America. Pipeline fracture experience, 1-21.

Chang, J., Xu, J.Q. and Mutoh, Y. (2006). A general mixed-mode brittle fracture criterion for cracked materials. Engineering Fracture Mechanics, 73(9), 1249-1263.

Erdogan, F. and Sih, G.C. (1963). On the crack extension in plates under plane loading and transverse shear. Journal of basic engineering, 85(4), 519-525.

Fu, G., Yang, W., Deng, W., Li, C.Q. and Setunge, S. (2019). Prediction of Fracture Failure of Steel Pipes With Sharp Corrosion Pits Using Time-Dependent Reliability Method With Lognormal Process. Journal of Pressure Vessel Technology, 141 (3), 031401 average shear stress yield Kucera, V. and Mattsson, E. , C.Q. and Mahmoodian, corrosion, Reliability En C.Q. and Melchers, R.
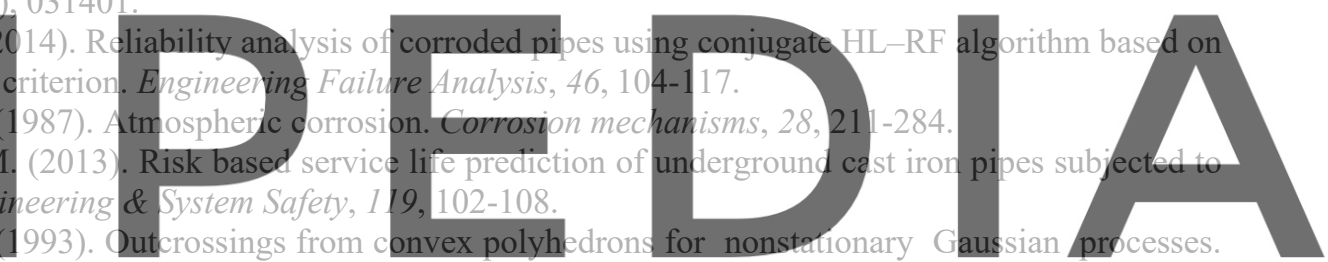
Journal of engineering mechanics, 119(11), 2354-2361.

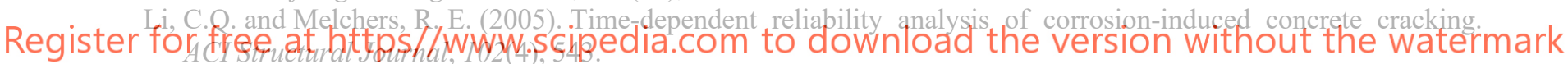

Li, C. Q., Fu, G. and Yang, W. (2016). Stress intensity factors for inclined external surface cracks in pressurised pipes. Engineering Fracture Mechanics, 165, 72-86.

Liu, A., Chen, K., Huang, X., Chen, J., Zhou, J. and Xu, W. (2019). Corrosion failure probability analysis of buried gas pipelines based on subset simulation. Journal of Loss Prevention in the Process Industries, 57, 25-33.

Mahmoodian, M. and Li, C.Q. (2016). Structural integrity of corrosion-affected cast iron water pipes using a reliability-based stochastic analysis method. Structure and Infrastructure Engineering, 12(10), 1356-1363.

Mahmoodian, M. and Li, C.Q. (2017). Failure assessment and safe life prediction of corroded oil and gas pipelines. Journal of Petroleum Science and Engineering, 151, 434-438.

Marshall, P. (2001). The residual structural properties of cast iron pipes: structural and design criteria for linings for water mains. UK Water Industry Research.

Melchers, R.E. (1999). Structural reliability analysis and prediction 2nd edition. John Wiley \& Sons, Chichester.

Rice, S.O. (1944). Mathematical analysis of random noise. Bell System Technical Journal, 23(3), 282-332.

Richard, H.A. (2001). Experimental and numerical simulation of mixed-mode crack growth. In Proceedings of the Sixth International Conference on Biaxial/Multiaxial Fatigue \& Fracture, 623-630.

Richard, H.A., Schramm, B. and Schirmeisen, N.H. (2014). Cracks on mixed mode loading-theories, experiments, simulations. International Journal of Fatigue, 62, 93-103.

Sadiq, R., Rajani, B. and Kleiner, Y. (2004). Probabilistic risk analysis of corrosion associated failures in cast iron water mains. Reliability Engineering \& System Safety, 86(1), 1-10.

Schöllmann, M., Richard, H. A., Kullmer, G. and Fulland, M. (2002). A new criterion for the prediction of crack development in multiaxially loaded structures. International Journal of Fracture, 117(2), 129-141.

Shiratori, M. and Miyoshi, T. (1986). Analysis of stress intensity factors for surface cracks subjected to arbitrarily distributed stresses. In Computational Mechanics' 86, 1027-1032. 
Wang, W., Li, C.Q. and Shi, W. (2019). Degradation of mechanical property of corroded water pipes after long service. Urban Water Journal. DOI: 10.1080/1573062X.2019.1687744.

Wang, W., Robert, D., Zhou, A. and Li, C. Q. (2018). Factors Affecting Corrosion of Buried Cast Iron Pipes. Journal of Materials in Civil Engineering, 30(11), 04018272.

Wang, W., Shi, W. and Li, C.Q. (2019). Time dependent reliability analysis for cast iron pipes subjected to pitting corrosion. International Journal of Pressure Vessels and Piping, 175, 103935.
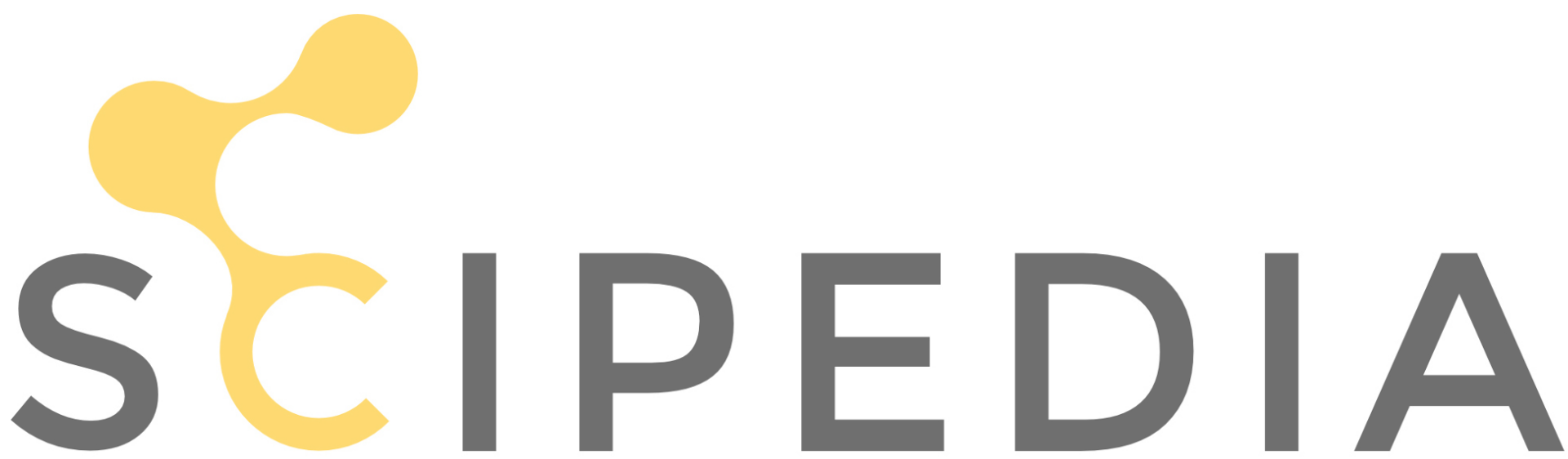

Register for free at https//www.scipedia.com to download the version without the watermark 